\title{
Diagnostics of macroscopic quantum states of Bose-Einstein condensate in double-well potential by nonstationary Josephson effect
}

\author{
E. D. Vo* \\ B.I. Verkin Institute for Low Temperature Physics and Engineering National \\ Academy of Sciences of Ukraine, Lenin av. 47 Kharkov 61103, Ukraine
}

(Dated: November 14, 2018)

\begin{abstract}
We propose a method of diagnostic of a degenerate ground state of Bose condensate in a double well potential. The method is based on the study of the one-particle coherent tunneling under switching the time-dependent weak Josephson coupling between the wells. We obtain a simple expression that allows to determine the phase of the condensate and the total number of the particles in the condensate from the relative number of the particles in two wells $\Delta n=n_{1}-n_{2}$ measured before the Josephson coupling is switched on and after it is switched off. The specifics of the application of the method in the cases of the external and the internal Josephson effect are discussed.

PACS numbers: 03.75.Fi, 05.30.Jp
\end{abstract}

Beginning from its first observation [1] the BoseEinstein condensation (BEC) of atoms in alkali metal vapours remains the source for new possibilities for the study of macroscopic quantum phenomena. One of these phenomena is the coherent tunneling of atoms between two coupled Bose condensates (BC) [2], that is analogous to the Josephson effect in superconductors. It is known 3] that for the case when the total number of atoms in the trap $N=n_{1}+n_{2}$ is conserved and the trap is symmetric relative two $\mathrm{BC}$ the average relative number of atoms $\overline{n_{1}-n_{2}}=\left\langle\Psi\left|\hat{n}_{1}-\hat{n}_{2}\right| \Psi\right\rangle$ is equal to zero in the ground state and in any excited state. Therefore, one can expect that in such a situation the study of nonstationary coherent tunneling (which is realized when one or several parameters of the system depends on time) is more informative for the diagnostics of the macroscopic wave functions of the condensates. In this case the average value of the relative number of the atoms $\overline{n_{1}-n_{2}}$ measured in the time $t_{0}$ is generally nonzero one and it depends on the history of the systems at all $t<t_{0}$. In this paper we show that nonstationary Josephson effect can be used for the diagnostics of a macroscopic state of $\mathrm{BC}$ and the total number of the atoms in the condensate.

We consider the simple model of coherent tunneling between two BC, described in [4] (see also references in it). The model is based on the two mode approximation, that implies that each of $N$ bosons can be in one of two states and the dynamical coupling between these state allows the bosons to jump from one state to the other. Such a model is applicable for a description of the external as well as the internal Josephson effect in Bose systems. The external Josephson effect [5] can be realized if the Bose gas is confined in a double-well trap and the tunneling between two wells is small. In this case two modes correspond to self consistent ground states in each well. The internal Josephson effect [ $\underline{6}$ can be realized in a Bose gas with two macroscopically occupied hyperfine

*Electronic address: vol@ilt.kharkov.ua states (e.g. the $\left|F=1, m_{F}=-1\right\rangle$ and $\left|F=2, m_{F}=1\right\rangle$ states of ${ }^{87} \mathrm{Rb}$ atoms). The dynamical coupling between the two states is settled by a resonant laser field applied to the system. At the beginning we specify the simplest case of the external Josephson effect at $T=0$.

The Hamiltonian of the symmetric two-mode model has the form

$$
H=H_{0}^{s}+H_{1}(t) \equiv \frac{K}{8}\left(\hat{n}_{1}-\hat{n}_{2}\right)^{2}-\frac{E_{J}(t)}{2}\left(a_{1}^{+} a_{2}+h . c\right),
$$

where $a_{i}^{+}\left(a_{i}\right)$ are the creation (annihilation) operators for the well $i$, and $\hat{n}_{i}=a_{i}^{+} a_{i}$ are the number operators. The parameter $K$ is determined by the interaction between the atoms in the well. Here we consider the case of $K>0$ that corresponds to the repulsive interaction. The value of $E_{J}$ is determined by the overlapping of the wave functions of two modes and it can be controlled by a variation of the height and (or) width of the barrier. For the external Josephson effect without loss of generality one can set the Josephson coupling $E_{J}(t)$ be real.

Let us consider the situation when the dynamical coupling between two condensates is switched on at $t=$ $t_{i}=0$ and switched off at $t=t_{f}$. At $t<0$ and $t>t_{f}$ the coupling parameter $E_{J}(t)=0$ and the occupation numbers operators $\hat{n}_{1}$ and $\hat{n}_{2}$ as well as the relative number operator $\hat{n}_{1}-\hat{n}_{2}$ commute with the Hamiltonian and do not depend on time. During the time when the coupling is switched on the operator $\hat{n}_{1}-\hat{n}_{2}$ is changed. Let at $t=0$ the wave function of the two mode Bose condensate is $\Psi(0)$ and at $t=t_{f}$ it becomes $\Psi\left(t_{f}\right)$. The task we consider is how to find the characteristics of the function $\Psi(0)$ from the measurements of the mean relative number $\left.\langle\Psi)\left|\hat{n}_{1}-\hat{n}_{2}\right| \Psi\right\rangle$. Let us specify the case of an odd total number of the particles (the case of an even $N$ is discussed below). At $N=2 M+1$ and $E_{j}=0$ the ground state of the Hamiltonian (1) is double-degenerate. The minimum of the energy equal to $K / 8$ is reached for the orthogonal states $\left|g_{1}\right\rangle=|M+1, M\rangle$ and $\left|g_{2}\right\rangle=|M, M+1\rangle$ as well as for an arbitrary superposition of these states $|g\rangle=a\left|g_{1}\right\rangle+b\left|g_{2}\right\rangle$ $\left(|a|^{2}+|b|^{2}=1\right)$. The state at $t=0$ can be parameterized 
as $|\Psi(0)\rangle=\cos (\theta / 2)|M+1, M\rangle+\sin (\theta / 2) e^{i \varphi}|M, M+1\rangle$ At $\theta \neq 0, \pi$ this is the entangled state. The angle $\theta$ is connected with the initial relative number by the relation

$$
\Delta n(0) \equiv\left\langle\Psi(0)\left|\hat{n}_{1}-\hat{n}_{2}\right| \Psi(0)\right\rangle=\cos \theta .
$$

Since this value does not depend on $\varphi$ the phase cannot be extracted from the result of measurements of the initial relative number. But the phase $\varphi$ is also an essential characteristics of the macroscopic state of the Bose condensate. In particular, the interference pattern emerging under an overlapping of two $\mathrm{BC}$ with the phases $\varphi_{1}$ and $\varphi_{2}$ is determined by its relative phase $\phi=\varphi_{1}-\varphi_{2}$. We will show that the value of $\varphi$ can be determined from the measurements of the final relative number $\Delta n_{f} \equiv\left\langle\Psi\left(t_{f}\right)\left|\hat{n}_{1}-\hat{n}_{2}\right| \Psi\left(t_{f}\right)\right\rangle$. To do this the amplitude of the Josephson coupling should be taken such a small that the strong inequality $N E_{J}^{\max } / K \ll 1$ be satisfied. Then at $0<t<t_{f}$ the system remains in the Fock regime. In this regime the dynamics of the system is realized mainly on the states for which $\left|\left\langle\Psi(t)\left|\hat{n}_{1}-\hat{n}_{2}\right| \Psi(t)\right\rangle\right| \leq 1$. Therefore to find the evolution of the function $\Psi$ one can use the basis $\left(\left|g_{1}\right\rangle,\left|g_{2}\right\rangle\right)$. Note that the reqime considered is the same as required for a realization of the Bose qubit [].

It is more convenient to use the unitary transformed basis of symmetric $|s\rangle=\left(\left|g_{1}\right\rangle+\left|g_{2}\right\rangle\right) / \sqrt{2}$ and antisymmetric $|a\rangle=\left(\left|g_{1}\right\rangle-\left|g_{2}\right\rangle\right) / \sqrt{2}$ states. In this basis the wave function of the $\mathrm{BC}$ reads as $\Psi(t)=s(t)|s\rangle+a(t)|a\rangle$ Using the nonstationary Schrödinger equation $i \hbar \dot{\Psi}=H \Psi$ one finds that the functions $s(t)$ and $a(t)$ satisfy the equations

$$
\begin{aligned}
& i \hbar \dot{s}=\frac{K}{8} s-\frac{E_{j}(t)(M+1)}{2} s, \\
& i \hbar \dot{a}=\frac{K}{8} a+\frac{E_{j}(t)(M+1)}{2} a .
\end{aligned}
$$

Integrating equations (3), (4) we find the mean value of the relative number at the time $t_{f}$

$$
\begin{array}{r}
\Delta n\left(t_{f}\right)=s^{*}\left(t_{f}\right) a\left(t_{f}\right)+c . c .=s^{*}(0) a(0) E^{-2 i \Omega}+c . c .= \\
=\cos \theta \cos (2 \Omega)-\sin \theta \sin (2 \Omega) \sin \varphi,(5)
\end{array}
$$

where $\Omega=(1 / 2 \hbar)(M+1) \int_{0}^{t_{f}} E_{J}\left(t^{\prime}\right) d t^{\prime}$.

Eq. (5) determines the relation between the measured quantity $\Delta n\left(t_{f}\right)$ and the parameter $\varphi$. One can see that for entangled initial states the relative number in a final state depends on the phase $\varphi$ and this phase can be found from the measurement of $\Delta n\left(t_{f}\right)$.

Thus, if one has a system in a reproducible (but unknown) initial state $|\Psi(0)\rangle$ the parameters $\theta$ and $\varphi$ that describe this state can be found from two sets of measurements of the relative number at $t=0$ and $t \geq t_{f}$ (under assumption that the total number of particles $N$ in the condensate is known). If the total number of the particles is unknown an additional set of measurements is required: the measurement of the final relative number occupation with another value of $t_{f}$. Using the results of three sets of measurements one can determine the initial state and find the total number of the particles in the condensate.

It is necessary to point out an essential restriction for the maximum value of $t_{f}-t_{i}$. In deriving (5) we do not take into account that the coupling between two condensates induces small (of order of $E_{J}(M+1) / K$ ) but nonzero occupation of the excited states $\left|e_{k}^{1}\right\rangle=$ $|M+k+1, M-k\rangle\left|e_{k}^{2}\right\rangle=|M-k, M+k+1\rangle$ (with $k>0)$. Due to such processes the phases of $s(t)$ and $a(t)$ are shifted from the values given by the solution of Eqs. (3), (4). If such a shift is of order of unity the relation (5) is not valid any more. Nevertheless, one can show that for $t_{f}-t_{i} \ll \hbar K /\left[E_{J}^{\max }(M+1)\right]^{2}$ the phase shifts are very small and Eq. (5) is applicable. The fulfillment of the mentioned restriction on the value of $t_{f}-t_{i}$ is required for the use of the method of the diagnostics proposed.

Let us now discuss the case of the $\mathrm{BC}$ with an even number of atoms. In the symmetric double-well trap the ground state of the condensate with $N=2 M$ is $|g\rangle=|M, M\rangle$. This state is nondegenerate and $\Delta n(0)=\Delta n\left(t_{f}\right)=0$. If initially the system in an excited state $|e\rangle=a|M+1, M-1\rangle+b|M-1, M+1\rangle$ then $\Delta n(0)=2\left(|a|^{2}-|b|^{2}\right)$ can be nonzero, but for $t_{f}-t_{i} \ll$ $\hbar K /\left[E_{J}^{\max }(M+1)\right]^{2}$ the difference $\Delta n\left(t_{f}\right)-\Delta n(0)$ is of order of $M E_{J}^{\max } / K \ll 1$ Such behavior are in difference with the case of odd $N$ when the change of $\Delta n$ can be order of unity. This feature can be used for determining the parity of the number of atoms in $\mathrm{BC}$. We point out again that this conclusion is for the symmetric relative two BC confining potential.

If the confining potential is an asymmetric one the Hamiltonian (1) is modified to

$$
\begin{gathered}
H_{1}=H_{0}^{a}+H_{1}(t) \\
\equiv \frac{K}{8}\left(\hat{n}_{1}-\hat{n}_{2}\right)^{2}-\Delta \mu\left(\hat{n}_{1}-\hat{n}_{2}\right)-\frac{E_{J}(t)}{2}\left(a_{1}^{+} a_{2}+h . c\right) .(6)
\end{gathered}
$$

One can see that if $\Delta \mu=K / 4$, the ground state of the system with an even number of the atoms $N=2 M$ is double-degenerate at $E_{J}=0$, and its wave function can be presented in the form $|g\rangle=a|M+1, M-1\rangle+b|M, M\rangle$. This situation is analogous to the symmetric case with odd $N$. The only difference is that the values of $\Delta n(0)$ and $\Delta n\left(t_{f}\right)$ given above are counted from $\Delta n=1$. Thus, under assumption that one can control the value of $\Delta \mu$ with the accuracy $|\Delta \mu-K / 4| \ll M E_{J}^{\max }$ the method of diagnostic of the ground state wave function and the total number of the atoms suggested is applicable for the BC with even $N$.

Hitherto we have discussed the case of the external Josephson effect. The case of the internal Josephson effect is also described by the equation (6) (in the rotating frame of reference) [4]. In this case the expression for the chemical potential $\Delta \mu$ reads as

$$
\Delta \mu=-\delta+\frac{4 N \pi \hbar^{2}}{m} \tilde{\eta}\left(a_{11}-a_{22}\right),
$$

where $\delta$ is the detuning of the laser field from the resonant frequency, $a_{11}$ and $a_{22}$, the s-wave scattering amplitudes 
of macroscopically occupied internal states $|1\rangle$ and $|2\rangle$, correspondingly. In a situation, where the value of the detuning can be varied smoothly, one can achieve the regime of the degenerate ground state of the Hamiltonian $H_{0}^{a}$ both for the even $\left(\delta=\delta_{e}\right)$ and for the odd $\left(\delta=\delta_{o}\right)$ number of atoms $N$. In such a regime one can apply the method of diagnostics of the initial state of BC proposed in this paper. We note that for the case of the internal Josephson effect the value of $\overline{\Delta n\left(t_{f}\right)}$ is just proportional to the expectation value of $M_{z}\left(t_{f}\right)$ - the projection of the magnetic momentum of the $\mathrm{BC}$ on the axis chosen. Therefore, using Eq. (5) one can determine the phase $\varphi$ and the total number of the atoms in the condensate from the measurement of $M_{z}\left(t_{f}\right)$.

I would like to acknowledge R.I.Shekhter, S.I.Shevchenko and D.V.Fil for the discussion of the results presented in this article. This work is supported by the INTAS grant No 01-2344.
[1] M. H. Anderson, J. R. Ensher, M. R. Matthew, C. E. Wieman, and E. A. Cornell, Science 269, 198 (1995); C. C. Bradley, C. A. Sackett, J. J. Tollett, and R. G. Hulet, Phys. Rev. Lett. 75, 1687 (1995); K. B. Davis, M. O. Mewes, M. R. Andrews, N. J. van Druten, D. S. Durfee, D. M. Kurn, and W. Ketterle, Phys. Rev. Lett. 75, 3969 (1995).

[2] G. J. Milburn, J. Corney, E. M. Wright, and D. F. Walls, Phys. Rev. A 554318 (1997); A. Smerzi, S. Fantoni, S. Giovanazzi, and S. R. Shenoy, Phys. Rev. Lett 79, 4950 (1997); S. Raghavan, A. Smerzi, S. Fantoni, and S. R. Shenoy, Phys. Rev. A 59, 620 (1999).

[3] S.Raghavan, A.Smerzi, and V.M.Kenkre, Phys. Rev. A 60, R1787 (1999).

[4] A.L.Leggett, Rev. Mod. Phys. 73, 307 (2001)
[5] J.Javanainen, Phys. Rev. Lett 57, 3164 (1986); F. Dalfovo, L. Pitaevskii, and S. Stringari, Phys. Rev A 54, 4213 (1996).

[6] C. J. Myatt, E. A. Burt, R. W. Ghrist, E. A. Cornell, and C. E. Wieman, Phys. Rev. Lett 78, 586 (1997); M. R. Matthews, D. S. Hall, D. S. Jin, J. R. Ensher, C. E. Wieman, and E. A. Cornell, F. Dalfovo, C. Minniti, and S. Stringari, Phys. Rev. Lett., 81, 243 (1998); D. S. Hall, M. R. Matthews, J. R. Ensher, C. E. Wieman, and E. A. Cornell, Phys. Rev. Lett., 81, 1539 (1998); D. S. Hall, M. R. Matthews, C. E. Wieman, and E. A. Cornell, Phys. Rev. Lett., 81, 1543 (1998);

[7] Z.-B. Chen and Y.-D. Zhang Phys. Rev. A 65, 022318 (2002) 